\title{
2.45GHZ, 3.7GHZ VE 5.8 GHZ FREKANSLARDA ÇALIŞAN ÜÇ-BANT YAMA ANTEN TASARIMI
}

\author{
Orhan ARMAĞAN*, Mesud KAHRIMAN
}

Süleyman Demirel Üniversitesi, Mühendislik Fakültesi, Elektonik ve Haberleşme Mühendisliği Bölümü, Isparta, Türkiye

\begin{tabular}{l}
\hline Anahtar Kelimeler \\
\hline Mikroşerit Anten \\
$\ddot{U} C$ Bant Anten \\
Yama Anten \\
$2.45 \mathrm{GHz}, 3.7 \mathrm{GHz}$ ve $5.8 \mathrm{GHz}$
\end{tabular}

Özet
Kullanımı gittikçe artan kablosuz ağve mobil haberleşme sistemlerinde, birden fazla
frekans bandında çalışacak anten birimlerine ihtiyaç duyulmaktadır. Bu çalışmada;
$2.45 \mathrm{GHz}, 3.7 \mathrm{GHz}$ ve $5.8 \mathrm{GHz}$ frekans bantlarında çalışan, mikroşerit yama anten
tasarımı anlatılmıştır. Bu antenin frekans bant genişlikleri sırasıla $110 \mathrm{MHz}$,
$220 \mathrm{MHz}$ ve $390 \mathrm{MHz}$ ve her üç frekansta da yansıma katsayıları $-27 \mathrm{~dB}$ civarında
ölçülmüştür. Bu antenin boyutları $52 \times 76 \times 1.6 \mathrm{~mm}^{3}$ 'dir. Anten tasarımı için HFSS
simülasyon programı kullanılmıştır. Tasarlanan anten çift taraflı bakır plaket
üzerinde basılarak $\mathrm{S}_{11}$ parametreleri Agilent FieldFox N9926A Network Analizör
cihazı ile ölçülmüştür. Ölçülen değerler ile simülasyon değerleri karşılaştırılmış ve
grafiksel olarak gösterilmiştir.

TRIPLE-BAND PATCH ANTENNA DESIGN WORKING IN 2.45GHZ,3.7GHZ AND 5.8GHZ FREQUENCIES

\begin{tabular}{|c|c|}
\hline Keywords & Abstract \\
\hline $\begin{array}{l}\text { Microstrip Antenna, } \\
\text { Triple Band Antenna, } \\
\text { Patch Antenna, } \\
2.45 \mathrm{GHz}, 3.7 \mathrm{GHz} \text { and } 5.8 \mathrm{GHz}\end{array}$ & $\begin{array}{l}\text { Increasing the use of wireless networks and mobile communication systems, the } \\
\text { antenna unit is required to operate in multiple frequency bands. In this study, } \\
\text { working in } 2.45 \mathrm{ghz}, 3.7 \mathrm{GHz} \text { and } 5.8 \mathrm{GHz} \text { frequency bands a antenna were designed. } \\
\text { This antenna's frequency bandwidths respectively } 110 \mathrm{MHz}, 220 \mathrm{MHz} \text { and } 390 \mathrm{MHz} \\
\text { is. The reflection coefficients in the around }-27 \mathrm{~dB} \text { at all three frequencies were } \\
\text { measured. The size of this antenna is } 52 \times 76 \mathrm{x} 1.6 \mathrm{~mm}^{3} \text {. This antenna is sized as a } \\
\text { parametric frequency specified in the HFSS program was simulated. This antenna } \\
\text { were printed on double-sided copper plate, and Antenna parameters were } \\
\text { measured with Aglent FieldFox N9926A Network Analyser. The values were } \\
\text { obtained with the simulation were compared with measured values and were } \\
\text { shown as graphically. }\end{array}$ \\
\hline
\end{tabular}

\section{Giriş}

Mikroşerit yama antenler, ilk defa 1953 y1lında keşfedilmesine ve 1955 yılında patenti alınmasına rağmen, 1970'li yıllarda dikkat çekerek popüler olmaya başladı. İlk etapta uçak, uzay mekiği, uydu ve füze uygulamaları radar sistemleri gibi, boyut, ağırlık, performans, montaj kolaylığı açısından kısıtlayıcı uygulamalarda, ardından mobil ve kablosuz haberleşme gibi ticari ve kamusal uygulamalarda, küçük profilli antenlere ihtiyaç duyulmaya başlanmıştı. Bu gereksinimi karşılayabilmek amacıyla mikroşerit yama antenler için geniş bir kullanım alanı doğmuştur. Bu süreçte bilimsel araştırmalar için de bir odak noktası haline gelmiştir.

Küçük profile sahip olması, tüm yüzeylere uyumlu basit yapıları, düşük maliyetle üretilebilmeleri, sağlam olmaları en önemli avantajlarıdır. Basit yapıları, dielektrik tabaka üzerine çeşitli geometrilerde 1şıma amaçlı bir yama ve dielektriğin alt yüzeyinde oluşturulan toprak zemin yamasından ibarettir. Yamanın beslenmesinde genellikle iletken mikroşerit hat veya yamanın uygun bir noktasından uygulanan prob kullanilır.

Mikroşerit yama antenlerin avantajlarının yanında düşük verimli, düşük güçlü olmaları, dar frekans band genişliğine sahip olmaları gibi önemli dezavantajları da mevcuttur.

$\mathrm{Bu}$ çalışmada HFSS simülasyon programı kullanılarak $2.45 \mathrm{GHz}, 3.7 \mathrm{GHz}$ ve $5.8 \mathrm{GHz}$ frekanslarında rezonansa gelen basit yapılı mikroşerit yama anten tasarımı anlatılmıştır. Tasarlanan antene ait $\mathrm{S}_{11}$ yansıma katsayısının simülasyon ve ölçüm sonuçları karşılaştırmalı

\footnotetext{
*ilgili yazar/Corresponding author: oarmagan@gmail.com, +90-246-441-4334
} 
olarak bir grafikte verilirken yine duran dalga oran1, toplam kazanç 1şıma örüntülerinin simülasyon sonuç grafikleri de gösterilmiştir.

\section{Bilimsel Yazın Taraması}

Mobil haberleşme ve kablosuz iletişimin yaygınlaşmasıyla farklı frekans bantlarında veri transferi ve haberleşme ihtiyaçları oluşmuştur. Bu nedenle farklı frekanslarda yani birden fazla frekans bantlarında çalışabilen çok bantlı ya da geniş bantlı anten tasarımları önem kazanmış, bu konuda birçok bilimsel çalışma yapılmıştır. Bu konuda yapılan çalışmalardan bazı örnekler aşağıda verilmiştir.

Huang ve arkadaşları üç bantlı biyotelemetri haberleşmesi için küçültmüş, vücuda yerleștirilebilir rectenna uygulaması üzerine çalışmışlardır. Antenin boyutları $10 \mathrm{~mm}$ x $10 \mathrm{~mm}$ x $2.54 \mathrm{~mm}$ 'dir. $5 \mathrm{k}$ ohm yük altında $433 \mathrm{MHz}$ çalışma frekansında, giriş gücü $11 \mathrm{~dB}$ olduğunda elde ettikleri dönüşüm verimi \%86dır. Yaptıkları antenin testlerini domuzun ön ayağından aldıkları et üzerinde gerçekleştirmişlerdir. $\mathrm{Bu}$ testler sonucunda elde ettikleri maksimum kazançlar $402 \mathrm{MHz}$ 'de $-7 \mathrm{~dB}, 433 \mathrm{MHz}$ 'de $-11 \mathrm{~dB}$ ve $2.45 \mathrm{GHz}$ 'de $15 \mathrm{~dB}^{\prime} \operatorname{dir}($ Huang et al., 2011).

Guatam ve arkadaşları 2013'de tasarladıkları CPW beslemeli ultra-geniş band mikroşerit antende yaptıkları parametrik ölçüm sonuçlarını şekildeki grafiklerle sunmuşlardır. Tasarladıkları anten prototipinin görüntüsü şekil 3.5 ve şekil 3.6 'da gösterilmiş olup, prototip $25 \times 25$ x $1.6 \mathrm{~mm}$ boyutlarında $2.6-13.04 \mathrm{GHz}(10.44 \mathrm{GHz})$ bir çalışma bant genişliği üzerinden iyi empedans eşleme, sürekli kazanç, istikrarlı ışıma desenlerine ulaşmışlardır (Gautam, Yadav, \& Kanaujia, 2013).

Malacooti ve arkadaşları 2015 yılında yaptıkları 2.45/5.8GHz'de çift bant çalışan mikroşerit anten çalışmasında $2.47 \mathrm{GHz}^{\prime} \mathrm{de}-22 \mathrm{~dB} \mathrm{~S}_{11}$ simülasyon ve 2.48GHz de $-22 \mathrm{~dB}_{11}$ ölçüm sonucu, 5.85GHz'de $-43 \mathrm{~dB}$ $\mathrm{S}_{11}$ simülasyon ve $5.88 \mathrm{GHz}$ 'de $-28 \mathrm{~dB} \mathrm{~S}_{11}$ ölçüm sonucu elde etmişlerdir. $\mathrm{Bu}$ anten yaklaşı $5 \times 5 \mathrm{~cm}$ boyutlarındadır. (Malakooti, Hayati, Fahimi, \& Afzali, 2015).

Karli ve Ammor, temelde tam toprak düzlemiyle dar band genişliğine sahip olan patch antende kısmi toprak düzlemi oluşturularak bant genişliğinin arttırılmasını sağladıklarını belirtmişlerdir. Aynı çalışmada patch anten üzerindeki yarıkların frekans, band genişliği ve S11 parametresi üzerine etkilerini incelemişler 2.45GHz'de $-28 \mathrm{~dB}$ ve $5.8 \mathrm{GHz}$ 'de $-25 \mathrm{~dB}$ civarında yansıma katsayısı elde etmişlerdir. Tasarladıkları anten $60 \times 70 \mathrm{~mm}$ boyutlarındadır (Karli \& Ammor, 2015).

Deepti Das Krishna ve arkadaşları çalışma frekansı $2.5 \mathrm{GHz}$ ile $4.5 \mathrm{GHz}$ arasında olan fraktal yapıda yarıklı anten tasarlamışlar ve yansıma katsayısını $2.5 \mathrm{GHz}$ 'de $24 \mathrm{~dB}$ olarak vermişlerdir. Önerdikleri antenin bant genişliği $1.57 \mathrm{GHz}$ olup, boyutları 33.5 x $28.5 \mathrm{~mm}^{2}$ 'dir. Maksimum kazanç 4.5 dBi 'dir (Krishna, Gopikrishna, Anandan, Mohanan, \& Vasudevan, 2008).
Zhu ve arkadașları, 2.4 / $5.8 \mathrm{GHz}$ ISM bantlarını kapsayan ve simülasyonda $-27 \mathrm{~dB}$ ve $-35 \mathrm{~dB}$ S11 değerleri gördükleri bir dual bant anten prototipi imal ederek 2.45 ve $5.8 \mathrm{GHz}$ frekansları için sırasıyla 115 ve 928MHz'lik bant genişlikleri ile 1.37 ve $4.37 \mathrm{dBi}$ kazanç değerleri elde etmişlerdir (Zhu, Guo, \& Wu, 2015).

Baytöre ve arkadaşları 2015 yılındaki çalışmalarında, taşınabilir kablosuz terminaller için yeni bir çift-bant MIMO anten dizisi önermişlerdir. Antenlerde 2.45 ve 5.8 GHz kablosuz uygulama için $10 \mathrm{~dB}$ return loss gereksinimini; $2.45 \mathrm{GHz}$ bandında $27 \mathrm{~dB}$ ve $5.8 \mathrm{GHz}$ bandında $20 \mathrm{~dB}$ üzeri izolasyonlar ile sağlamışlardır (Baytore, Palandoken, Kaya, \& Zoral, 2015).

Zhou ve arkadaşları geniş bandlı dairesel polarizasyon için düzgün altıgen yapıda CPW beslemeli bir geniş bant slot anten tasarlamışlar ve bu antende $1.8 \mathrm{GHz}$ ile $4.6 \mathrm{GHz}$ arasındaki geniş frakans aralığında kesintisiz $10 \mathrm{~dB}$ altında yansıma katsayısı elde etmişlerdir (Zhou, Li, Wang, Feng, \& Liu, 2011).

\section{Materyal ve Yöntem}

$2.45 \mathrm{GHz}, 3.7 \mathrm{GHz}$ ve $5.8 \mathrm{GHz}$ frekanslarında çalışan üç bant anten tasarımı yapılırken Ansoft HFSS programı kullanılmıştır. Tasarlanan antenin hesaplamaları, bağıl dielektrik katsayısı 3.7, kayıp tanjant değeri 0.045 olan çift taraflı bakır plaket malzemesi üzerinden gerçekleştirilmiştir. Antenin dıştan dışa boyutları $51.6 \mathrm{~mm} \times 75.5 \mathrm{~mm}$, plaket kalınlığı ise $1.6 \mathrm{~mm}$ 'dir.

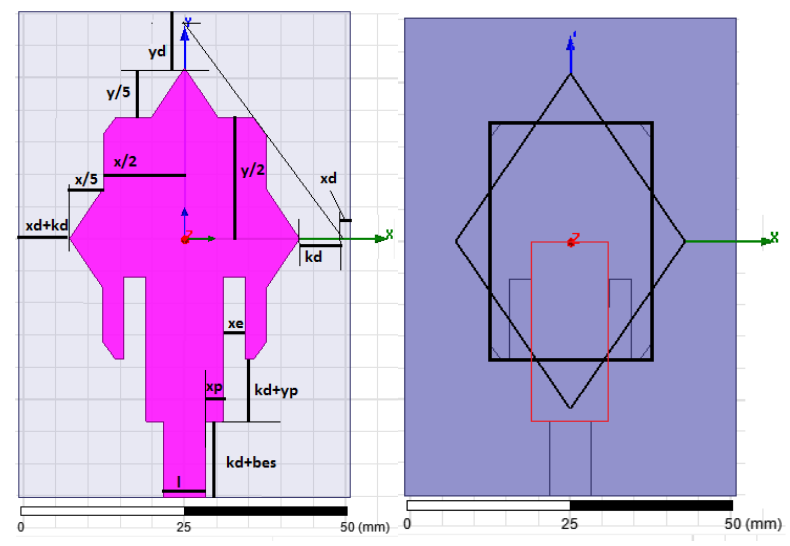

Şekil 1. (a) Anten geometrisi ve değișken isimleri ve (b) geometrinin çıkarılmasındaki aşamalar.

Anten tasarlanırken klasik yama anten üzerine bir dikdörtgen ve bir eşkenar dörtken eklenip iki yarık çıkarılması ve dikdörtgen yama köşelerinin kesilmesiyle elde edilmiş bir geometriye sahiptir. $\mathrm{Bu}$ anten geometrisi ve çıkarılma aşamaları Şekil 1'de, basılmış anten görünüşü de Şekil 2'de gösterilmiştir. $\mathrm{Bu}$ antenin frekans- $\mathrm{S}_{11}$ değişimi simülasyon ve ölçüm sonuçları Şekil 3'de gösterilmiştir. Verilen ölçüm sonuçları Agilent FieldFox N9926A Network Analizör cihazı ile ölçülmüştür. İlk oluşturulan yama antenin dikdörtgen şeklindeki orta kısmının boyutları $\mathrm{x}$ ve $\mathrm{y}$ olarak alınmıştır ve bu değerlerin değişiminin anten $\mathrm{S}_{11}$ parametresine etkisi karşılaştırmalı olarak Şekil 4'de ve Şekil 5'de gösterilmiştir. Anten geometrisinde kullanılan değişkenler ve değerleri Tablo 1'de gösterilmiştir. 
Tablo 1: Anten geometrisi değişkenleri ve değerleri

\begin{tabular}{|c|c|c|c|c|c|}
\hline Değişken & $\begin{array}{c}\text { Değer } \\
(\mathrm{mm})\end{array}$ & Değişken & $\begin{array}{c}\text { Değer } \\
(\mathrm{mm})\end{array}$ & Değişken & $\begin{array}{c}\text { Değer } \\
(\mathrm{mm})\end{array}$ \\
\hline \$x & 25.3 & \$y & 37 & \$bes & 5 \\
\hline \$xd & 6.4 & \$yd & 9.75 & \$I & 6.5 \\
\hline \$xp & 2.75 & \$yp & 3 & Boy & 75.5 \\
\hline \$xe & 3.5 & \$kd & 6.75 & En & 51.6 \\
\hline
\end{tabular}

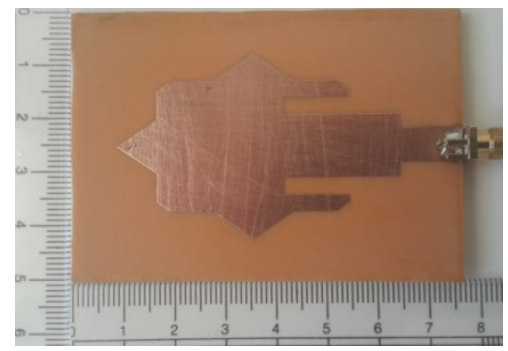

Şekil 2. Önerilen ve ölçüm yapılmış olan anten görünüşü

\section{Araştırma Bulguları}

Şekil 1'de gösterilen anten geometrisi, HFSS programında çizilip geometrideki boyutların çizelge 1'deki değerlere ayarlanmasının ardından, 2GHz$6.5 \mathrm{GHz}$ aralığı için şekillerde gösterilen grafiklerdeki yansıma katsayısını belirten $S_{11}$ parametresi, duran dalga oranı, ışıma örüntüleri elde edilmiştir. Tasarlanan antenin $2 \mathrm{GHz}-6.5 \mathrm{GHz}$ aralığı için yansıma katsayısının simülasyon ve ölçüm değerini gösteren grafik Şekil 3'de x ve y parametrelerine göre değișen frekans-S11 grafikleri de Şekil 4 ve Şekil 5'de gösterilmiştir.

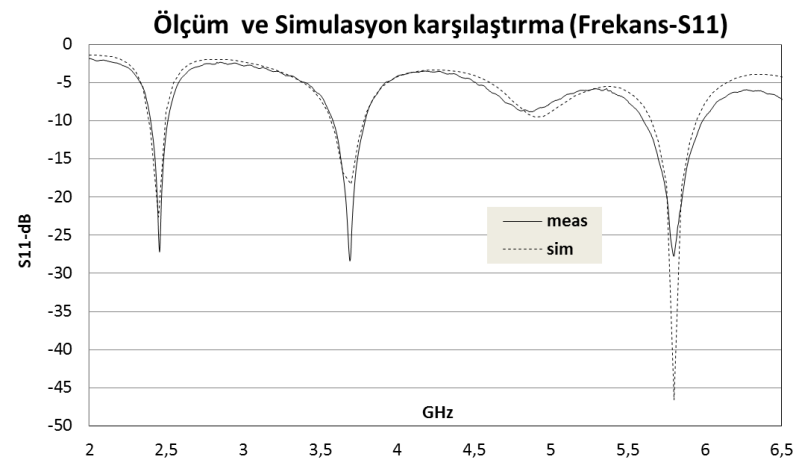

Şekil 3. Önerilen antenin Frekans- $\mathrm{S}_{11}$ değişiminin ölçüm ve simülasyon sonuçlarının karşılaştırılması

Şekil 3'deki ölçüm değerlerine göre $-10 \mathrm{~dB}$ altında kalan frekans değerlerine göre $2.40-2.51 \mathrm{GHz}$ aralığında $110 \mathrm{MHz}, 3.58-3.80 \mathrm{GHz}$ aralığında $220 \mathrm{MHz}, 5.60-5.99 \mathrm{GHz}$ aralığında da $390 \mathrm{MHz}$ frekans bant genişlikleri ölçülmüştür.

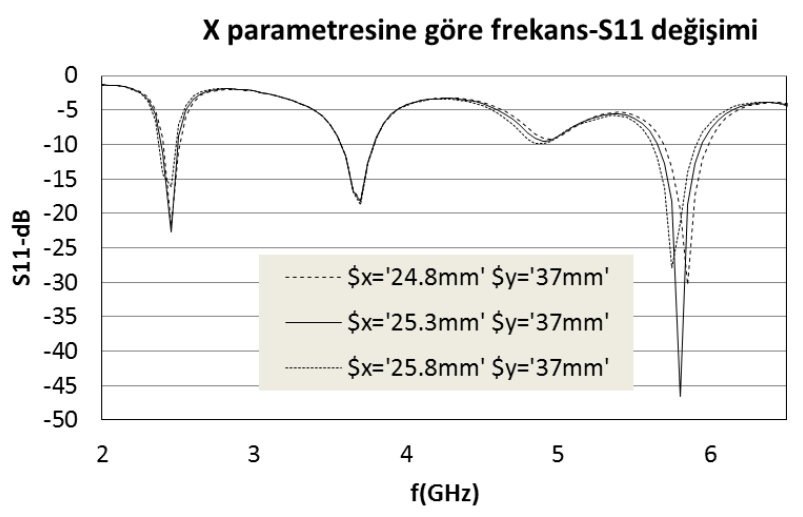

Şekil 4. Önerilen antenin değişen $x$ parametresine göre frekans$\mathrm{S}_{11}$ simülasyon sonuçları

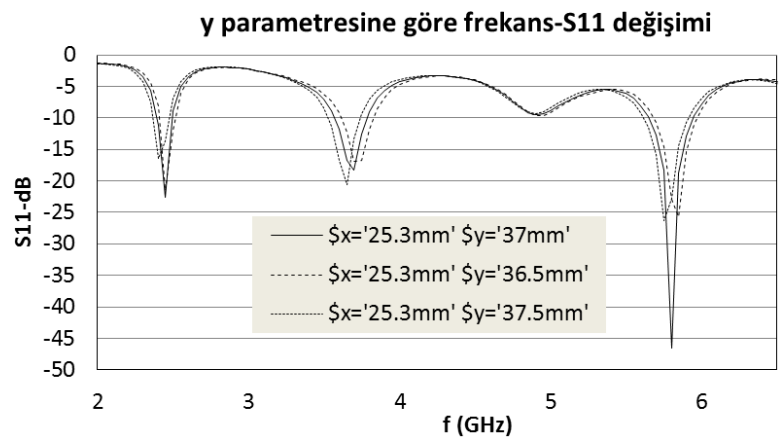

Şekil 5. Önerilen antenin değişen y parametresine göre frekans-S ${ }_{11}$ simülasyon sonuçları

Şekil 6'da her üç frekans için 2 boyutlu toplam kazanç ışıma örüntülerinin Theta $=0 / 30 / 60 / 90^{\circ}$ için değişimleri gösterilmiştir.

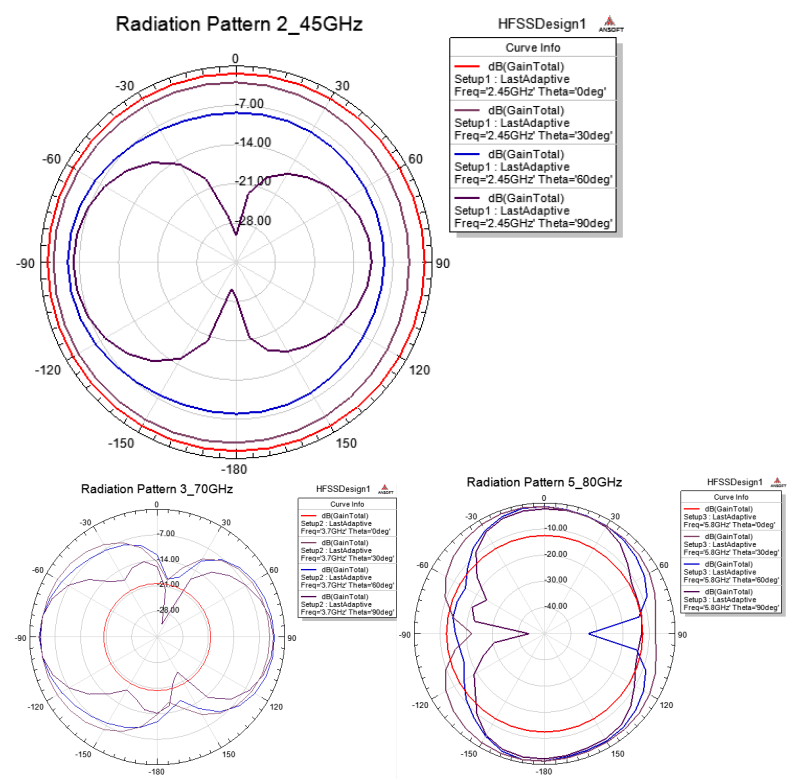

Şekil 6. 2.45/3.7/5.8GHz frekansları için Theta=0/30/60/90 için toplam kazanç ışıma örüntüleri

Şekil 7'de tasarladığımız antene ait frekans-Duran Dalga Oranı (VSWR) grafiği verilmektedir. Duran dalga oranı 2'nin altında olması istenen değer olup S11'in -10dB altında kalan frekans değerleri için duran dalga oranının da 2'nin altında olduğu grafikten okunabilir. Grafikte 5 
farklı sonuç verilmiştir. Bu sonuçlar antenin $x-y$ değer değişimlerine bağlı olarak sunulmuştur.

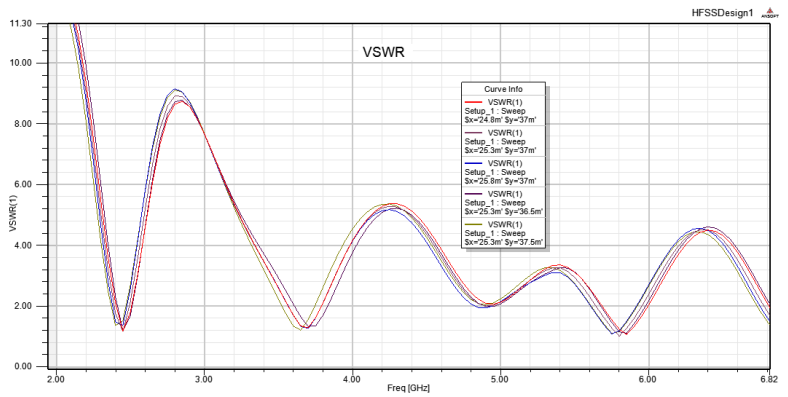

Şekil 7. $x$ ve y parametrelerinin değişimine göre FrekansDuran Dalga Oranı (VSWR) değișimi

Tasarlamış olduğumuz antene ait üç boyutlu ışıma örüntüleri Şekil 8, Şekil 9 ve Şekil 10 incelendiğinde $2.45 \mathrm{GHz}$ 'de $-1.24 \mathrm{dBi}, 3.7 \mathrm{GHz}$ 'de $-2.59 \mathrm{dBi}$ ve $5.8 \mathrm{GHz}$ 'de -0.45dBi kazançlara sahip olduğu görülmektedir.

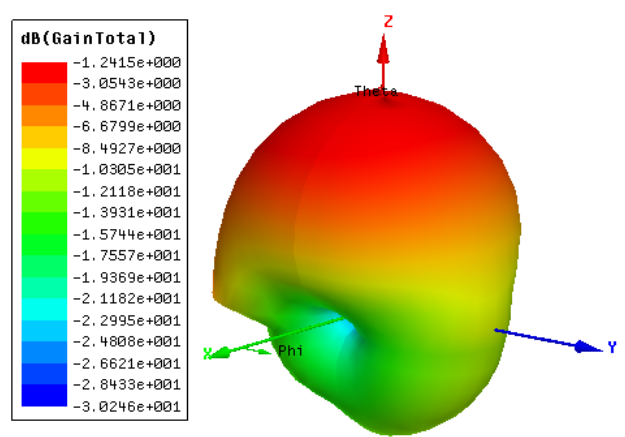

Şekil 8. Tasarlanan antenin $2.45 \mathrm{GHz}$ için dB olarak Toplam Kazancın üç boyutlu ıșıma örüntüsü
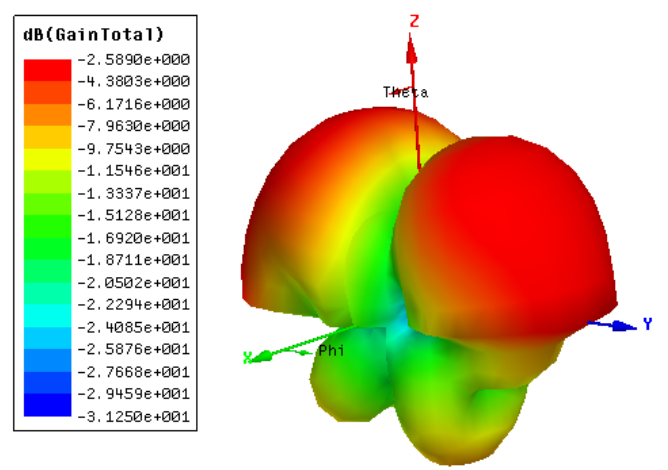

Şekil 9. Tasarlanan antenin 3.7GHz için dB olarak Toplam Kazancın üç boyutlu ışıma örüntüsü

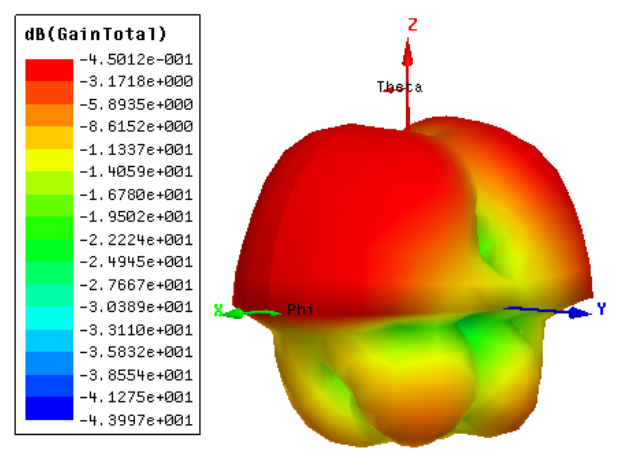

Şekil 10. Tasarlanan antenin 5.8GHz için dB olarak Toplam Kazancın üç boyutlu ışıma örüntüsü

\section{Sonuç ve Tartışma}

Yapılan çalışmada $2.45 \mathrm{GHz}, \quad 3.7 \mathrm{GHz}$ ve $5.8 \mathrm{GHz}$ frekanslarında üç-bant çalışabilen bir mikroşerit yama anten tasarımı gerçekleştirilmiştir. Tasarımı gerçekleştirilen antenin boyutları $51.6 \mathrm{~mm}$ x $75.5 \mathrm{~mm}$ x $1.6 \mathrm{~mm}$ 'dir. Simülasyon sonuçları ve ölçüm sonuçları verilen antenin üç bantta birden $-25 \mathrm{~dB}$ altında $S_{11}$ ölçüm değerine sahip olması daha önce yapılan benzer antenlere olan önemli bir üstünlüğüdür. Tasarlanan antenin sadece teoride kalmayı simülasyon sonuçlarının ölçüm sonuçlarıyla karşılaştırılması uygulama anlamında önemlidir.

Tablo 2: Tasarlanan antenin ölçüm sonuçlarının farklı çalışmalar ile karşılaştırılması

\begin{tabular}{|c|c|c|c|c|}
\hline & & \multicolumn{3}{|c|}{ S11 @Çalışma frekansları(ölçüm) } \\
\hline yazar & Boyut (mm) & $\mathrm{F} 1(\mathrm{GHz}) / \mathrm{BG}$ & $\mathrm{F} 2(\mathrm{GHz}) / \mathrm{BG}$ & $\mathrm{F} 3(\mathrm{GHz}) / \mathrm{BG}$ \\
\hline \multirow{2}{*}{ Huang et al, 2011} & \multirow{2}{*}{$10 \times 10 \times 2.54$} & $-12 \mathrm{~dB} @ 2.45$ & $-25 \mathrm{~dB} @ 0.466$ & \multirow{2}{*}{$\cdots$} \\
\hline & & $70 \mathrm{MHz}$ & $113 \mathrm{MHz}$ & \\
\hline \multirow{2}{*}{ Malakooti et al., 2015} & \multirow{2}{*}{$50 \times 50$} & $-30 \mathrm{~dB} @ 2.55$ & \multirow{2}{*}{$\cdots$} & $-28 \mathrm{~dB} @ 5.85$ \\
\hline & & $1 \mathrm{GHz}$ & & $2 \mathrm{GHz}$ \\
\hline \multirow{2}{*}{ Karli \& Ammor, 2015} & \multirow{2}{*}{$60 \times 70$} & $-13 \mathrm{~dB} @ 2.73$ & \multirow{2}{*}{$\cdots$} & $-29 \mathrm{~dB} @ 7.11$ \\
\hline & & $40 \mathrm{MHz}$ & & $880 \mathrm{MHz}$ \\
\hline \multirow{2}{*}{ Zhu et al, 2015} & \multirow{2}{*}{ Cap $=61.6$} & $-27 \mathrm{~dB} @ 2.45$ & \multirow{2}{*}{$\cdots$} & $-35 \mathrm{~dB} @ 5.78$ \\
\hline & & $115 \mathrm{MHz}$ & & $928 \mathrm{MHz}$ \\
\hline \multirow{2}{*}{ Baytōre ve ark, 2015} & \multirow{2}{*}{$15 \times 50 \times 1.6$} & $-17 \mathrm{~dB} @ 2.45$ & \multirow{2}{*}{$\cdots$} & $-15 \mathrm{~dB} @ 5.7$ \\
\hline & & $550 \mathrm{MHz}$ & & $650 \mathrm{MHz}$ \\
\hline \multirow{2}{*}{ Baytöre ve ark, 2015} & \multirow{2}{*}{$125 \times 100 \times 1.6$} & $-13 \mathrm{~dB} @ 2.5$ & $-24 \mathrm{~dB} @ 4.55$ & $-35 \mathrm{~dB} @ 5.8$ \\
\hline & & $100 \mathrm{MHz}$ & $250 \mathrm{MHz}$ & $350 \mathrm{MHz}$ \\
\hline \multirow{2}{*}{ Baytöre ve ark., 2015} & \multirow{2}{*}{$63 \times 50 \times 1.6$} & $-23 \mathrm{~dB} @ 2.6$ & $-22 \mathrm{~dB} @ 4.6$ & $-34 d B @ 5.95$ \\
\hline & & $500 \mathrm{MHz}$ & $300 \mathrm{MHz}$ & $1400 \mathrm{MHz}$ \\
\hline \multirow{2}{*}{ Mevcut Çalışma } & \multirow{2}{*}{$52 \times 75 \times 1.6$} & $-27 \mathrm{~dB} @ 2.45$ & $-28 \mathrm{~dB} @ 3.7$ & $-27 \mathrm{~dB} @ 5.8$ \\
\hline & & $110 \mathrm{MHz}$ & $220 \mathrm{MHz}$ & $390 \mathrm{MHz}$ \\
\hline
\end{tabular}

Benzetim ve ölçüm sonuçları verilen anten ile geçmişte yapılmış benzer antenlerin boyut, yansıma katsayısı, çalışma frekansı ve çalışma frekans bant genişliklerinin ölçüm sonuçlarının karşılaştırılması Tablo 2'de gösterilmiştir.

Tablo 2 incelendiğinde antenlerin farklı alanlarda üstünlük sağladığı gözlenmektedir. Önerilen antenin üç çalışma frekansında birden ölçüm değerlerinde frekans kayması bulunmaması diğer antenlere karşı bir üstünlüktür. Bant genişliklerinin dar kalmasının avantaj olabileceği göz önünde bulundurulmalıdır. Çalışmada tasarlanan anten $2 \mathrm{GHz}-6.5 \mathrm{GHz}$ frekans aralığında 2.45/3.7/5.8 GHz frekanslarında olmak üzere hem WLAN hem de WiMAX işlemleri için uygun olan üç farklı çalışma frekansında ışıma yapma özelliğine sahip özgün bir antendir.

\section{Kaynaklar}

Baytore, C., Palandoken, M., Kaya, A., \& Zoral, Y. (2015). Compact multiband MIMO antenna designs and analysis for 2.4/5.2/5.8 GHz WLAN MIMO systems. Paper presented at the Signal Processing and Communications Applications Conference (SIU), 2015 23th.

Gautam, A. K., Yadav, S., \& Kanaujia, B. K. (2013). A CPW-fed compact UWB microstrip antenna. 
IEEE Antennas and Wireless Propagation Letters, 151-154.

Huang, F.-J., Lee, C.-M., Chang, C.-L., Chen, L.-K., Yo, T.-C., \& Luo, C.-H. (2011). Rectenna application of miniaturized implantable antenna design for triple-band biotelemetry communication. Antennas and Propagation, IEEE Transactions on, 59(7), 2646-2653.

Karli, R., \& Ammor, H. (2015). Rectangular Patch Antenna for Dual-Band RFID and WLAN Applications. Wireless Personal Communications, 1-13.

Krishna, D. D., Gopikrishna, M., Anandan, C., Mohanan, P., \& Vasudevan, K. (2008). CPW-fed Koch fractal slot antenna for WLAN/WiMAX applications. IEEE Antennas and Wireless Propagation Letters, 7, 389-392.

Malakooti, S.-A., Hayati, M., Fahimi, V., \& Afzali, B. (2015). Generalized dual-band branch-line coupler with arbitrary power division ratios. International Journal of Microwave and Wireless Technologies, 1-9.

Zhou, S.-W., Li, P.-H., Wang, Y., Feng, W.-H., \& Liu, Z.-Q. (2011). A CPW-Fed Broadband Circularly Polarized Regular-Hexagonal Slot Antenna With L-Shape Monopole. IEEE ANTENNAS AND WIRELESS PROPAGATION LETTERS, 10.

Zhu, X., Guo, Y., \& Wu, W. (2015). A Novel Dual-band Antenna for Wireless Communication Applications. 